\title{
Alterations in the Blood Velocity Profile Influence the Blood Flow Response during Muscle Contractions and Relaxations
}

\author{
Takuya OSADA 1,2 and Göran RÅDEGRAN2,3 \\ 1Department of Sports Medicine for Health Promotion, Tokyo Medical University, Tokyo, 160-8402 Japan; 2The Copenhagen Muscle \\ Research Centre, University of Copenhagen, Rigshospitalet, DK-2100 Copenhagen Ø, Denmark; and 3Department of Cardiology, The \\ Heart and Lung Division, Lund University Hospital, S-221 85 Lund, Sweden
}

\begin{abstract}
The present study examined the influences of the muscle contraction (MCP) and relaxation (MRP) phases, as well as systole and diastole, on the blood velocity profile and flow in the conduit artery at different dynamic muscle contraction forces. Eight healthy volunteers performed one-legged dynamic knee-extensor exercise at work rates of 5, 10, 20,30, and $40 \mathrm{~W}$ at 60 contractions per minute. The time- and space-averaged, amplitude-weighted, mean $\left(V_{\text {mean }}\right)$ and maximum $\left(V_{\max }\right)$ blood flow velocities were continuously measured in the common femoral artery during the cardiosystolic (CSP) and cardiodiastolic (CDP) phases during MCP and MRP, respectively. The $V_{\max } l$ $V_{\text {mean }}$ ratio was used as a flow profile index where a ratio of approximately $(\sim) 1$ indicates a "flat" velocity profile, and a ratio significantly greater than (>>) 1 indicates a "parabolic" velocity profile. At rest, a "steeper" parabolic velocity profile was found
\end{abstract}

during the $\operatorname{CDP}$ (ratio: $1.75 \pm 0.06$ ) than during the CSP (ratio: $1.31 \pm 0.02)$. During the MRP of exercise, the $V_{\text {max }} / V_{\text {mean }}$ ratio shifted to be less steep $(p<0.05)$ than at rest during the CDP (ratio: 1.41-1.54) at 5, 10, 20,30, and $40 \mathrm{~W}$; whereas it was slightly higher $(p<0.05)$ at 30 and $40 \mathrm{~W}$ than at rest during the CSP (ratio: 1.43-1.46). During the MCP, the parabolic blood velocity profile was enhanced $(p<0.05)$ at higher contraction forces, $\geq 20 \mathrm{~W}$ during the $\operatorname{CDP}$ (ratio: 2.15-2.52) and $\geq 30 \mathrm{~W}$ during the CSP (ratio: 1.49-1.77), potentially because of a greater retrograde flow component. A higher blood flow furthermore appeared during the MRP compared to during the MCP, coinciding with a greater uniformity of the red blood cells moving at higher blood velocities during the MRP. Thus part of the difference in the magnitude of blood flow during the MRP vs. MCP may be due to the alterations of the blood velocity flow profile.

Key words: rheological blood flow profile, blood velocity, exercise hyperemia, Doppler ultrasound.

$\mathrm{T}_{\mathrm{h}}$ he red blood cell velocity distribution in a conduit artery at rest varies between the cardiosystolic and the cardiodiastolic phase [1-4]. In the carotid and femoral arteries at rest, it has previously been demonstrated that the blood velocity profile during the cardiosystolic phase is less "steep" and somewhat more "flat" compared to the "steeper" parabolic velocity profile during the cardiodiastolic phase [4-6]. During dynamic exercise, the distribution of red blood cells moving at different velocities in a conduit artery may furthermore be altered because of variations in the intramuscular pressure, induced by the muscle contractions and relaxations, and its temporal relation to the perfusion pressure, cardiac cycle, and state of vasodilatation.

Considerable work has been performed in the area of the influence of dynamic exercise on the magnitude of blood flow in conduit arteries, and blood flow is known to increase linearly with exercise intensity [7-9]. It is further known that most blood flow occurs during the muscle relaxation phase and that muscle blood flow may be imped- ed during the muscle contraction phase [10]. Moreover, alterations in the femoral artery blood velocity flow profile during the cardiosystolic and cardiodiastolic phases have previously been observed at different work rates for consecutive rhythmic muscle contraction-relaxation duty cycles [11]. These findings may be because the various red blood cells moving in conduit arteries are differently influenced by the cardiac cycle during repetitive muscle contraction-relaxation duty cycles. However, precise measurements of the blood velocity profile and its influence on blood flow in relation to muscle contractions and relaxations during different cardiac phases have previously not been performed. This is of importance because the blood velocity flow profile may be disturbed by the muscle contraction force and will thus consequently influence the blood flow response during the muscle contraction $v s$. the relaxation phase.

Therefore a lack of knowledge exists with regard to the blood velocity distribution and its profile for each muscle contraction and relaxation phase, respectively, during the

Received on Dec 17, 2005; accepted on May 15, 2006; released online on May 20, 2006; doi:10.2170/physiolsci.RP002905 Correspondence should be addressed to: Takuya Osada, Department of Sports Medicine for Health Promotion, Tokyo Medical University, 6-1-1 Shinjuku, Shinjuku-ku, Tokyo, 160-8402 Japan. Phone: +81-3-5379-4339, Fax: +81-3-3226-5277, E-mail: DENTACMAC@aol.com 
cardiac cycle. A further understanding on how the blood flow velocity profile is altered during dynamic exercise in relation to the state of muscle contraction and relaxation and of the cardiac cycle may furthermore improve our knowledge about the control of exercise hyperemia, how it may be impaired in states of cardiovascular disease and how it may be optimized to enhance peak performance.

The hypothesis investigated in the present study was therefore that the blood velocity profile and consequently the flow, besides being influenced by the dynamic muscle contractions and relaxations at different exercise intensities, also may be influenced by the cardiosystolic and cardiodiastolic phases as a result of alterations in the blood velocity profile. Therefore as a direct continuation of our previous study [11], the present study reanalyzed the blood velocity profile and flow in the common femoral artery during the cardiosystolic and cardiodiastolic phases, at rest, and during the muscle contraction and relaxation phases of one-legged dynamic knee-extensor exercise at incremental work rates, previously recorded by Osada and Rådegran [11].

\section{METHODS}

Subjects. Eight healthy male volunteers with a mean \pm SE (range) age of $26.6 \pm 1.4$ (22-36) years, height of 183 $\pm 2(174-190) \mathrm{cm}$, and body weight of $77.3 \pm 2.9$ (64-90) $\mathrm{kg}$ participated in the experiments. The subjects participated in exercise varying from daily activities to regular endurance training and had no previous history of cardiovascular disease, hypertension, or anemia. Furthermore, they were examined by ultrasound, and no existence of arterial stenosis or atherosclerosis in the femoral artery was found, neither were varicose vein, edema, or arterial pulse in differences found in either leg, and no symptom of intermittent claudication. The subjects who volunteered were informed about the experimental procedures, the potential risks, and the discomfort, also that they could withdraw from the experiments anytime with no consequences. All eight volunteers participated after providing signed informed consents, and the experiments were carried out with approval by the Ethical Committees of Copenhagen and Frederiksberg (KF-01-013/96).

Experimental protocol. Before the experiments, all subjects were familiarized with the one-legged dynamic knee-extensor exercise (DKE) model [7, 12]. They trained at 60 contractions per minute $(\mathrm{cpm})$ at work rates of 5, 10, 20,30 , and $40 \mathrm{~W}$ until they were comfortable and could fully relax the hamstring muscles. This ensured that the work was being performed solely by the knee extensors of the quadriceps muscle group. With the thigh horizontal, the lower leg was moved up to a knee angle of $\sim 150$ degrees, where 180 degrees corresponds to a fully extended knee. The specific loads of 5, 10, 20,30, and $40 \mathrm{~W}$ corresponded to applied weights of $0.083,0.167,0.333,0.5$, and $0.667 \mathrm{~kg}$, respectively. The work rate was calculated according to the dynamic knee-extensor ergometer model $[7,12]$, defined as work rate $(W)=$ (contraction rate $[\mathrm{cpm}] / 60 \mathrm{~s}) \times($ distance of a knee-extensor revolution $[6$ $\mathrm{m}]) \times\left(\right.$ load weight $\left.[\mathrm{kg}] \times 9.81\left[\mathrm{~m} / \mathrm{s}^{2}\right]\right)$.

Peripheral arterial blood pressure (BP) was monitored continuously with a finger-cuff photoplethysmography device (FinapresTM, Ohmeda 2300, Englewood, NJ) placed on the middle finger of the left hand. The finger cuff was maintained next to the inguinal ligament in a fixed position at the level of the femoral artery. Mean arterial BP was calculated by integrating the BP curve over time. The kicking muscle force was measured with a strain gauge. The muscle force curve was used to represent the oscillations in intramuscular pressure, since a good correlation between muscle force and intramuscular pressure within the knee extensor muscle group previously has been demonstrated during DKE $[8,10,13]$. The electromyography (EMG) activity of the vastus lateralis was additionally recorded. The signals of all parameters were continuously recorded for approximately $4 \mathrm{~min}$ at steady-state DKE and stored in a computer using a MacLab data acquisition system (Chart v.3.5.7 software, ADInstruments, Sydney, Australia). The experimental protocols were performed on the same day. It was verified that a sufficient amount of time was allowed between the knee-extensor exercise bouts for blood flow to return to resting control levels. Belts attached to the seat fixed the upper body and both thighs. The femoral artery blood velocity measurements were performed by Doppler ultrasound for approximately $4 \mathrm{~min}$; following this a steady state was reached after $3 \mathrm{~min}$ of DKE [10,14], as previously described [8]. The contraction rhythm was maintained by following the pace of a visible and audible metronome and by watching the contraction rate displayed in real time on a monitor.

Blood velocity measurements with Doppler ultrasound. Doppler ultrasound is a noninvasive technique that provides high temporal and spatial measurements of the red blood cell velocity distribution in conduit blood vessels. The source of the signal producing the Doppler shift frequency is the "red blood cells" and their velocity and direction of movement. The blood velocity measured by Doppler ultrasound consequently reflects the hemodynamic characteristics of the red blood cells [5]. With regard to the laminar, nonpulsatile, steady-state flow of a Newtonian fluid in a straight rigid cylindrical tube, it is known that a flat velocity profile at a proximal site near a tube entrance progressively evolves to a parabolic velocity profile downstream at a distal site as a result of alterations in the boundary layer [5, 15-17]. Possible downstream flow profile changes from the inlet were, however, not accounted for in the present study because they involved a non-Newtonian fluid [18] with a pulsatile arterial blood velocity profile. 
Fig. 1. Analysis of $V_{\max }$ and $V_{\text {mean }}$ for the cardiosystolic and cardiodiastolic phases during the muscle contraction and muscle relaxation phases during one-legged dynamic knee-extensor exercise. The average maximum $\left(V_{\max }\right)$ and mean $\left(V_{\text {mean }}\right)$ blood velocity, muscle force, electromyography (EMG), and blood pressure (BP) during steady-state DKE at $20 \mathrm{~W}$ and 60 contractions per minute in one subject. $V_{\max }$ and $V_{\text {mean }}$ during the cardiosystolic and cardiodiastolic phases were measured continuously in parallel with the BP curve during the muscle contraction and muscle relaxation phases determined from the EMG and the muscle force curve. The blood velocity fluctuated in relation to the state of vasodilation and the muscle contraction-relaxation duty cycle (indicated by the oscillations in muscle force). Four variations (A-D) in the coupling between the BP curve and the state of contraction and relaxation were indicated, as previously described [10]: (A) the cardiosystolic phase during muscle contraction, (B) the cardiodiastolic phase during muscle contraction, (C) the cardiosystolic phase during muscle relaxation, and (D) the cardiodiastolic phase during muscle relaxation. $V_{\text {max }}$ and $V_{\text {mean }}$ were determined as the "average" transient maximum (outer envelope) and mean (amplitude-weighted, time-and-spatial averaged) angle-corrected blood velocity values, respectively, for the cardiosystolic and cardiodiastolic phases. The cardiosystolic (systole) and the cardiodiastolic (diastole) phases are indicated in the graph. The black thin line indicates $V_{\max }$. The gray thick line indicates $V_{\text {mean }}$. The formation of the blood velocity profile and flow was modified by the intramuscular pressure, as indicated by the muscle force curve, and the superimposed influence of the BP in relation to the

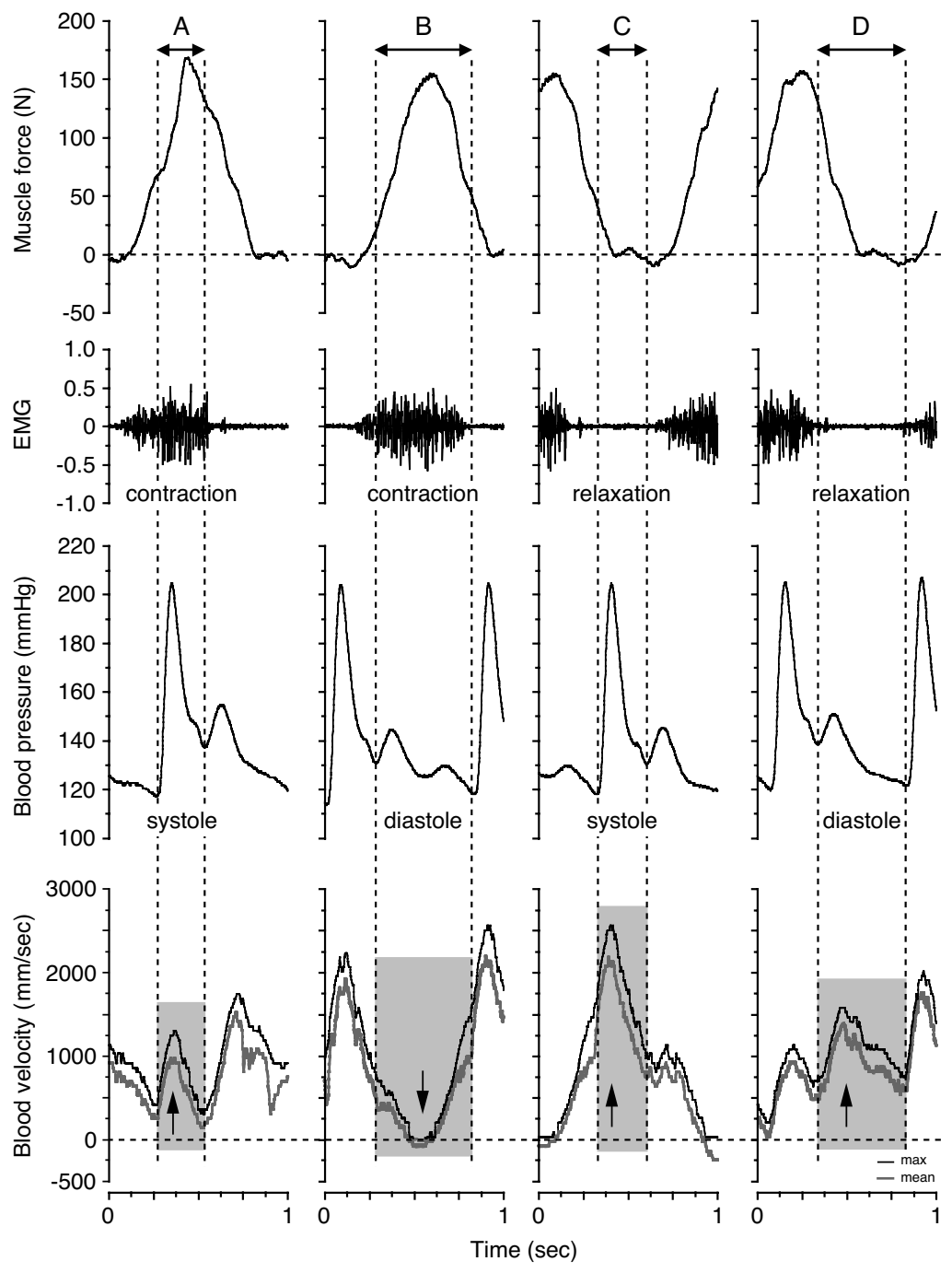
cardiosystolic and cardiodiastolic phases. The arrow down $(\downarrow)$ and up $(\uparrow)$ indicates the influence on the blood velocity and flow, depending on the magnitude of, and temporal relation between, the intramuscular pressure and the arterial BP, respectively.

The procedures for blood velocity and flow measurements in the common femoral artery have previously been validated and shown to produce accurate absolute values at rest and during DKE [8]. The equipment used was a Doppler ultrasound (model CFM 800, Vingmed Sound, Horten, Norway) equipped with an annular phased array transducer (Vingmed Sound) probe (11.5 mm diameter) operating at an imaging frequency of $7.5 \mathrm{MHz}$ and variable Doppler frequencies of 4.0-6.0 MHz (high-pulsed repetition frequency mode, $4-36 \mathrm{kHz}$ ). The angle-corrected, time and space-averaged, and amplitude-weighted mean $\left(V_{\text {mean }}\right)$ and maximum $\left(V_{\max }\right.$; outer envelope) blood flow velocities, respectively, were measured. $V_{\text {mean }}$ was defined by averaging the mean blood velocity trace $[8,9$, 19]. $V_{\max }$ was defined as the maximum outer envelope or peak blood velocity [20-26]. The $V_{\max }$ obtained in the present study was expressed as the blood velocity measured at the center of the vessel. Each blood velocity parameter was measured in relation to the BP curve.
Vessel location. The site for vessel diameter determination and blood velocity measurements in the common femoral artery was distal to the inguinal ligament, approximately one to two $\mathrm{cm}$ above the bifurcation into the superficial and profunda femoral branch $[8,14]$. This position was chosen to minimize any turbulence from the bifurcation and influence of blood flow from the inguinal region. Furthermore, the arterial diameter at this site is unaffected by the distal muscle contractions and relaxations. The blood velocity measurements were performed with the probe at an insonation angle as low as physically possible and always below 60 degrees [27]. The mean value of the probe angle in the present study was $45.5 \pm 0.8$ degrees and remained constant throughout the experiments for the individual subjects. Special care was taken to ensure that the insonation angle did not vary and that the sample volume was precisely positioned in the center of the vessel and adjusted to cover the width of the diameter. In the present study, the average width of the circular sam- 
Table 1. Average $V_{\max }$ and $V_{\text {mean }}$ at rest and during one-legged dynamic knee-extensor exercise for the cardiosystolic and cardiodiastolic phases during muscle contraction and muscle relaxation phases. The average maximum $\left(V_{\text {max }}\right)$ and mean $\left(V_{\text {mean }}\right)$ blood velocity increased $(p<0.05)$, respectively, for each consecutive exercise intensity. $V_{\max }$ was larger $(p<0.05)$ than $V_{\text {mean }}$ in the cardiosystolic phase during the muscle contraction phase at 30 and $40 \mathrm{~W}$ and during the muscle relaxation phase at $10-$ $40 \mathrm{~W}$, as well as in the cardiodiastolic phase during the muscle contraction phase at $10-40 \mathrm{~W}$ and the muscle relaxation phase at 5-40 W. $V_{\max }$ and $V_{\text {mean }}$ were higher in the muscle relaxation phase than in the muscle contraction phase during the cardiosystolic phase at 20-40 W and during the cardiodiastolic phase at 10-40 W, respectively. Significant difference $(p<0.05)$; (a) $V_{\text {max }}$ and $V_{\text {mean }}$ at each work rate $v s$. at rest; (b) $V_{\text {max }} v s$. $V_{\text {mean }}$ at rest and at each work rate, respectively; (c) $V_{\text {max }}$ and $V_{\text {mean }}$ during the muscle relaxation phase vs. during the muscle contraction phase. ${ }^{*} T h e$ values at rest were shared with those in the previous report [11]. The values are expressed as mean \pm SE.

\begin{tabular}{|c|c|c|c|c|c|c|}
\hline & \multicolumn{3}{|c|}{ Cardiosystolic phase } & \multicolumn{3}{|c|}{ Cardiodiastolic phase } \\
\hline & $V_{\text {mean }}(\mathrm{mm} / \mathrm{s})$ & $V_{\max }(\mathrm{mm} / \mathrm{s})$ & Sampling numbers & $V_{\text {mean }}(\mathrm{mm} / \mathrm{s})$ & $V_{\max }(\mathrm{mm} / \mathrm{s})$ & Sampling numbers \\
\hline${ }^{*}$ Rest & $162 \pm 17$ & $213 \pm 26^{b}$ & $60 \pm 12$ & $36 \pm 3$ & $63 \pm 5^{b}$ & $60 \pm 12$ \\
\hline \multicolumn{7}{|c|}{ Muscle contraction phase } \\
\hline $5 \mathrm{~W}$ & $527 \pm 82 a$ & $698 \pm 102 a$ & $53 \pm 11$ & $209 \pm 39 a$ & $312 \pm 41 a$ & $58 \pm 9$ \\
\hline $10 W$ & $643 \pm 79 a$ & $860 \pm 84 a$ & $55 \pm 10$ & $268 \pm 32^{a}$ & $400 \pm 32^{a, b}$ & $52 \pm 9$ \\
\hline $20 W$ & $654 \pm 89 a$ & $890 \pm 98 a$ & $61 \pm 7$ & $226 \pm 39 a$ & $397 \pm 40 \mathrm{a}, \mathrm{b}$ & $47 \pm 9$ \\
\hline $30 \mathrm{~W}$ & $744 \pm 80 a$ & $1,050 \pm 90 a, b$ & $55 \pm 6$ & $296 \pm 34 a$ & $541 \pm 38 a, b$ & $55 \pm 8$ \\
\hline $40 \mathrm{~W}$ & $678 \pm 81 a$ & $1,046 \pm 93 a, b$ & $34 \pm 8$ & $283 \pm 26^{a}$ & $548 \pm 30^{a, b}$ & $32 \pm 6$ \\
\hline \multicolumn{7}{|c|}{ Muscle relaxation phase } \\
\hline $5 \mathrm{~W}$ & $578 \pm 69 a$ & $779 \pm 83 a$ & $73 \pm 6$ & $266 \pm 34 a$ & $381 \pm 40 a, b$ & $57 \pm 6$ \\
\hline $10 \mathrm{~W}$ & $781 \pm 76^{a}$ & $1,040 \pm 80^{a, b}$ & $58 \pm 7$ & $371 \pm 32^{a, c}$ & $524 \pm 35^{a, b, c}$ & $53 \pm 8$ \\
\hline $20 \mathrm{~W}$ & $1,072 \pm 98 a, c$ & $1,429 \pm 115^{a, b, c}$ & $68 \pm 7$ & $563 \pm 36 a, c$ & $787 \pm 48 \mathrm{a}, \mathrm{b}, \mathrm{c}$ & $60 \pm 6$ \\
\hline $30 \mathrm{~W}$ & $1,189 \pm 74 a, c$ & $1,665 \pm 88 \mathrm{a}, \mathrm{b}, \mathrm{c}$ & $64 \pm 6$ & $708 \pm 51 \mathrm{a}, \mathrm{c}$ & $1,055 \pm 64 a, b, c$ & $64 \pm 6$ \\
\hline $40 W$ & $1,329 \pm 114 \mathrm{a}, \mathrm{c}$ & $1,907 \pm 163 a, b, c$ & $38 \pm 8$ & $884 \pm 55^{a, c}$ & $1,294 \pm 75^{a, b, c}$ & $36 \pm 8$ \\
\hline
\end{tabular}

pling volume was $11 \mathrm{~mm}$. The Doppler ultrasound system measured the blood velocity profile within this sampling volume.

Calculation of blood flow. The systolic and diastolic diameter size of the femoral artery was measured on a monitor in relation to the ECG. The mean vessel diameter was calculated in relation to the temporal duration of the BP curve: ([systolic diameter value $\times 1 / 3]+[$ diastolic diameter value $\times 2 / 3]$ ] [8]. The diameter measurements were obtained under perpendicular insonation. Signal interference from blood turbulence near the vascular wall was reduced with a low-velocity rejection filter. Femoral artery blood flow (FBF) was calculated by multiplying the cross-sectional area (area $\left.=\pi \times[\text { diameter } / 2]^{2}\right)(\mathrm{CSA})$ of the femoral artery with the angle corrected, time and space averaged, and amplitude-(signal intensity) weighted blood velocity $\left(V_{\text {mean }}\right.$ or $\left.V_{\text {max }}\right)$; where $\mathrm{FBF}_{\text {mean }}=V_{\text {mean }} \times \mathrm{CSA} \times 6 \times 10^{4}(\mathrm{ll}$ min) and $\mathrm{FBF}_{\max }=V_{\max } \times \mathrm{CSA} \times 6 \times 10^{4}(\mathrm{l} / \mathrm{min})$. The constant $6 \times 10^{4}$ is the conversion factor from $\mathrm{m} / \mathrm{s}$ to $\mathrm{l} / \mathrm{min}$ [8]. Because the femoral arterial diameter has been found not to change from rest to DKE $[8,9,23,28,29]$, the diameter measurements at rest were used for the calculation of FBF during both rest and DKE.

$V_{\text {max }} / V_{\text {mean }}$ ratio for the cardiosystolic and cardiodiastolic phases during the muscle contraction and muscle relaxation phases. The velocity profile index, expressed as the $V_{\max } / V_{\text {mean }}$ ratio [30], was determined for both the cardiosystolic and cardiodiastolic phases. The cardiosystolic phase started at the upstroke after minimum BP and ended before the second dicrotic BP notch. The cardiodiastolic phase was defined between the notch before the second dicrotic BP notch and the minimum BP before the upstroke (Fig. 1). Changes in the velocity profile were estimated from changes in the $V_{\max } / V_{\text {mean }}$ ratio. For a parabolic-like velocity profile, the $V_{\max } / V_{\text {mean }}$ ratio is significantly greater than $(>>)$ 1.0. Thus a greater increase in $V_{\max }$ compared to $V_{\text {mean }}$ implies a steeper parabolic-like profile. In contrast, for a flat plug-like velocity profile the $V_{\max } / V_{\text {mean }}$ ratio is approximately equal to $(\sim) 1.0$ [30]. The velocity profiles during the cardiosystolic and cardiodiastolic phases were evaluated during both the muscle contraction and relaxation phases (Fig. 1). The muscle contraction and relaxation phases were differentiated from the elecromyography (EMG) activity of the vastus lateralis [12].

Intraobserver variability. Before the experiments were pursued, extensive operator training was performed with the Doppler ultrasound technique to minimize any intraobserver variability during rest and DKE. At rest, the coefficients of variation determined from repeated blood velocity measurements were $3.4 \pm 0.3 \%$. During exercise, the coefficients of variation for the repeated blood velocity measurements were $<5 \%$ [14], which correspond to the oscillations in blood flow that occur because of variations in intramuscular pressure and its relation to the cardiac cycle and BP phases [10].

Statistical analysis. Parametric statistics were used for data analysis (i.e., a multiple analysis of variance for repeated measures and Fisher's significant difference post hoc tests when comparing more than two groups over time; a paired $t$-test when comparing but two groups). A $p$ 
Fig. 2. The $V_{\text {max }} / V_{\text {mean }}$ ratio for the cardiosystolic and cardiodiastolic phases at rest and during onelegged dynamic knee-extensor exercise during the muscle contraction and relaxation phases. A higher $(p<0.01) V_{\text {max }} / V_{\text {mean }}$ ratio was found during the cardiodiastolic compared to during the cardiosystolic phase at rest $(*)$ and during exercise $(*)$ during the muscle contraction phase, but not during the muscle relaxation phase. During the muscle contraction phase, the $V_{\text {max }} / V_{\text {mean }}$ ratios were higher $(p<0.01)$ than at rest during the cardiosystolic phase $(£)$ at 30

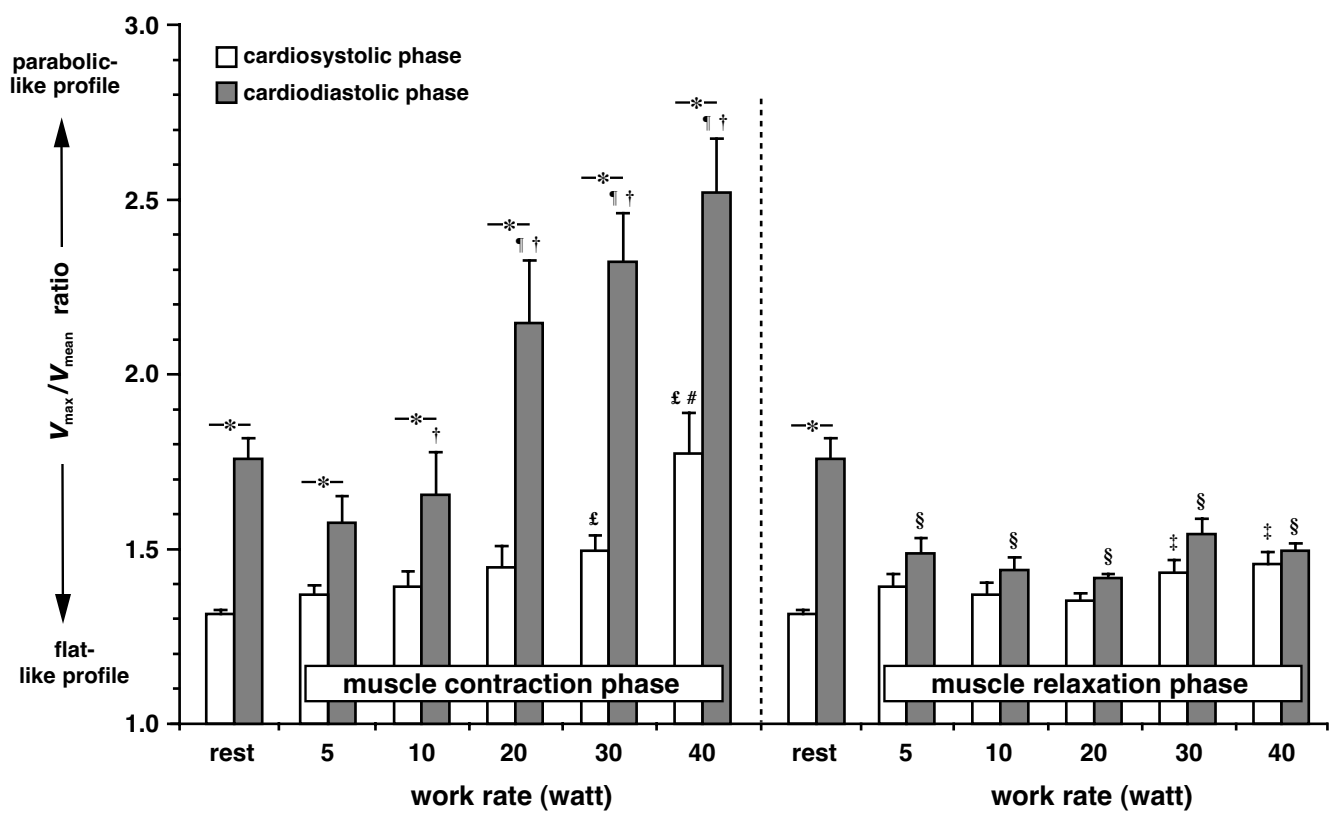

and $40 \mathrm{~W}$ and during the cardiodiastolic phase (T) at 20,30, and $40 \mathrm{~W}$. During the muscle relaxation phase, the $V_{\text {max }} / V_{\text {mean }}$ ratios were lower $(p<0.05)$ than at rest during the cardiodiastolic phase $(\S)$ at $5,10,20,30$, and $40 \mathrm{~W}$. Significant difference $(p<$ $0.05)$; the $V_{\text {max }} / V_{\text {mean }}$ ratio for the cardiosystolic (£) and the cardiodiastolic (T) phase at various work rates during muscle contraction vs. at rest. The $V_{\text {max }} / V_{\text {mean }}$ ratio for the cardiosystolic $(\ddagger)$ and the cardiodiastolic (§) phase at each work rate during muscle relaxation $v s$. at rest. The $V_{\text {max }} / V_{\text {mean }}$ ratio for the cardiosystolic (\#) and the cardiodiastolic ( $\dagger$ ) phase at various work rates during muscle contraction vs. during muscle relaxation. The values are expressed as mean $\pm \mathrm{SE}$.

value $<0.05$ was considered statistically significant. A $p=$ NS indicates not statistically significant. The values are mean \pm SE.

\section{RESULTS}

The femoral arterial diameter at rest was $10.9 \pm 0.3 \mathrm{~mm}$. The average $V_{\max }$ and $V_{\text {mean }}$ at rest and during DKE for the cardiosystolic and cardiodiastolic phases during the muscle contraction and relaxation phases are shown in Table 1 . The $V_{\max } / V_{\text {mean }}$ ratios at rest and during DKE are shown in Fig. 2. At rest, the $V_{\max } / V_{\text {mean }}$ ratio was $\sim 1.3$ and $\sim 1.8$ during the cardiosystolic and cardiodiastolic phases, respectively, as previously reported [11].

For the muscle contraction phase, the $V_{\max } / V_{\text {mean }}$ ratio was higher $(p<0.05)$ at 30 and $40 \mathrm{~W}$ than at rest during the cardiosystolic phase, and higher $(p<0.05)$ at 20,30 , and $40 \mathrm{~W}$ than at rest during the cardiodiastolic phase. During the muscle relaxation phase, the $V_{\max } / V_{\text {mean }}$ ratio was higher $(p<0.05)$ at 30 and $40 \mathrm{~W}$ than at rest during the cardiosystolic phase, whereas the $V_{\max } / V_{\text {mean }}$ ratio was lower $(p<0.05)$ at $5,10,20,30$, and $40 \mathrm{~W}$ compared to at rest during the cardiodiastolic phase. Furthermore, the $V_{\max } / V_{\text {mean }}$ ratio was higher $(p<0.05)$ during muscle contraction than during muscle relaxation at 10,20,30, and $40 \mathrm{~W}$ for the cardiodiastolic phase and at $40 \mathrm{~W}$ during the cardiosystolic phase. Moreover, the $V_{\max } / V_{\text {mean }}$ ratio was lower $(p<0.05)$ during the cardiosystolic phase than during the cardiodiastolic phase both at rest and during mus- cle contraction, but not during muscle relaxation (Fig. 2).

The $\mathrm{FBF}_{\max }$ and $\mathrm{FBF}_{\text {mean }}$, as well as the mean "arterial" $\mathrm{BP}$, during the cardiosystolic and cardiodiastolic phases at rest and during DKE for the muscle contraction and muscle relaxation phases are shown in Fig. 3. $\mathrm{FBF}_{\max }$ was higher $(p<0.05)$ than $\mathrm{FBF}_{\text {mean }}$ during the cardiosystolic phase, at 30 and $40 \mathrm{~W}$ during the muscle contraction phase, and at $10,20,30$, and $40 \mathrm{~W}$ during the muscle relaxation phase. They showed a tendency to be higher ( $p=$ NS) at the other work rates during both the muscle contraction and relaxation phases, respectively. $\mathrm{FBF}_{\max }$ was higher $(p<0.05)$ than $\mathrm{FBF}_{\text {mean }}$ during the cardiodiastolic phase at $10,20,30$, and $40 \mathrm{~W}$ during the muscle contraction phase, and at $5,10,20,30$, and $40 \mathrm{~W}$ during the muscle relaxation phase. A positive linear relationship $(p<$ $0.01 ; r, 0.972-0.999)$ for $\mathrm{FBF}_{\text {max }}$ and $\mathrm{FBF}_{\text {mean }} v s$. the incremental work rate was found during muscle relaxation for both the cardiosystolic and cardiodiastolic phase, respectively. $\mathrm{FBF}_{\text {max }}$ and $\mathrm{FBF}_{\text {mean }}$, respectively, were higher $(p<0.05)$ during the cardiosystolic than during the cardiodiastolic phase during both the muscle contraction and relaxation phases, respectively.

\section{DISCUSSION}

The present study shows that the blood velocity profile in the conduit femoral artery differs between the muscle contraction and the muscle relaxation phase in relation to the cardiac cycle. The blood velocity profile is less steep 

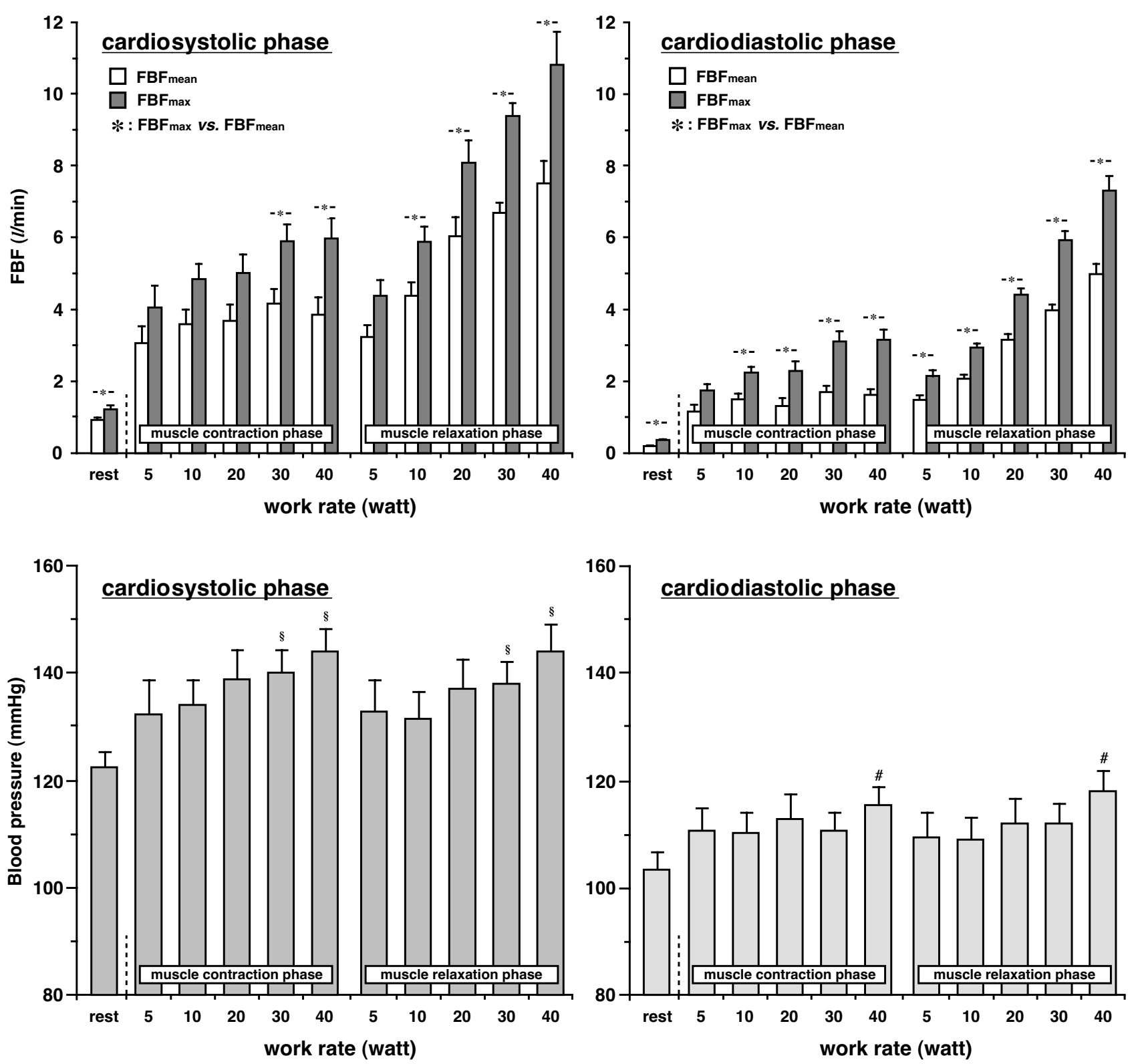

Fig. 3. FBF and BP for the cardiosystolic and cardiodiastolic phases at rest and during one-legged dynamic knee-extensor exercise during the muscle contraction and relaxation phases. Mean and maximum femoral artery blood flow (FBF) values increased linearly $(p<0.01 ; r, 0.972-0.999)$ with work rate during both the cardiosystolic and cardiodiastolic phases during muscle relaxation, but not to the same extent during muscle contraction. The regression lines during the cardiosystolic and cardiodiastolic phases during the muscle relaxation phase reflect the FBF value at a given work rate (WR) and phase; i.e., $\mathrm{FBF}_{\text {max-systole-relax }}(l / \mathrm{min})=3.937+0.179 \times \mathrm{WR}$;

and somewhat more flat-like in the muscle relaxation phase during both the cardiosystolic and cardiodiastolic phases, compared to the steeper and somewhat more parabolic-like velocity profile during the muscle contraction phase, especially as it coincides with the cardiodiastolic phase. Such alterations in the blood velocity profile may consequently be of physiological importance by altering
$\mathrm{FBF}_{\text {mean-systole-relax }}=3.083+0.118 \times \mathrm{WR} ; \mathrm{FBF}_{\text {max-diastole-relax }}=$ $1.433+0.148 \times W R ; F F_{\text {mean-diastole-relax }}=1.056+0.099 \times$ WR. Significant difference $(p<0.05)$; ${ }^{*} \mathrm{FBF}_{\max } v s$. $\mathrm{FBF}_{\text {mean }}$. The averaged blood pressure (BP) during the cardiosystolic phase during both the muscle contraction and muscle relaxation phases was significantly higher $(p<0.05)$ at 30 and 40 $\mathrm{W}$ than at rest $(\S)$. The averaged BP during the cardiodiastolic phase during both the muscle contraction and muscle relaxation phases was significantly higher $(p<0.05)$ at $40 \mathrm{~W}$ than at rest $(\#)$. The values are expressed as mean \pm SE.

the blood flow response to exercise and thus influencing performance.

\section{Alteration of the blood velocity profile during the muscle contraction and muscle relaxation phases in relation to the cardiac cycle}

The features of blood velocity and flow distribution 


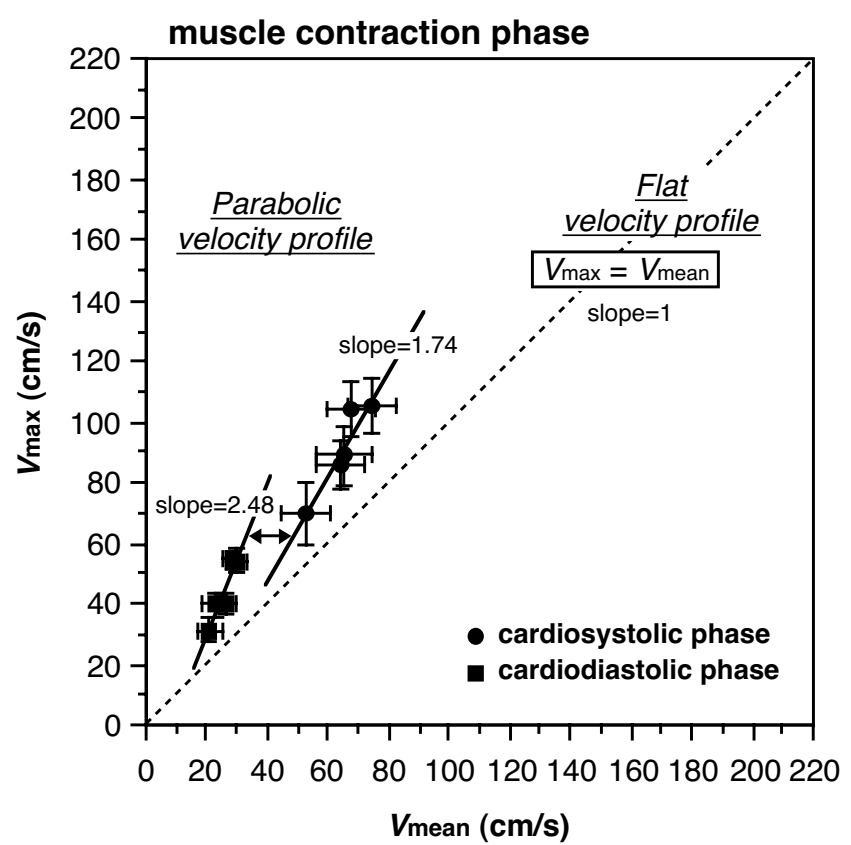

Fig. 4. Relationship between $V_{\max }$ and $V_{\text {mean }}$ for the cardiosystolic and cardiodiastolic phases during the muscle contraction and relaxation phases. There was a positive linear correlation between $V_{\max }$ and $V_{\text {mean }}$ for both the cardiosystolic (circles) and cardiodiastolic (squares) phases during the muscle contraction and muscle relaxation phase. The dotted line shows the line of identity $\left(V_{\max }=V_{\text {mean }}\right.$, slope $\left.=1\right)$, which defines a total flat velocity profile. During muscle contraction, the slope of the regression line "differed" $(p<0.05)$ markedly between the

during rhythmic thigh muscle contractions for consecutive contraction-relaxation duty cycles have previously been assessed by the use of $V_{\text {max }}, V_{\text {mean }}$, and the $V_{\text {max }} / V_{\text {mean }}$ ratio [11]. The separate effect of the muscle contraction and relaxation phases on the alterations of the rheological blood flow profile in relation to the cardiac cycle, however, was not addressed in the previous study [11]. In the present study, the previous recordings of Osada and Rådegran [11] were reanalyzed to address this specific issue. The $V_{\text {max }} / V_{\text {mean }}$ index ratio, previously used to describe the blood velocity profile, was in the present study found to vary with work rate during the cardiosystolic and cardiodiastolic phases during the muscle contraction and muscle relaxation phases (Fig. 2). During the muscle contraction phase at higher contraction forces such as $30-40$ $\mathrm{W}$, the $V_{\max } / V_{\text {mean }}$ ratio shifted toward a "steeper" and more parabolic-like velocity profile during both the cardiosystolic and cardiodiastolic phases, but it was most enhanced during the cardiodiastolic phase.

However, during the cardiodiastolic phase of the muscle relaxation phase, the $V_{\max } / V_{\text {mean }}$ ratio shifted toward a less steep and somewhat more flat-like velocity profile for each work rate $(5-40 \mathrm{~W}) . V_{\text {mean }}$ tended to be more similar to $V_{\max }$ during the muscle relaxation phase during both the cardiosystolic and the cardiodiastolic phase. At higher work rates, such as at $30-40 \mathrm{~W}$, during the cardiosystolic

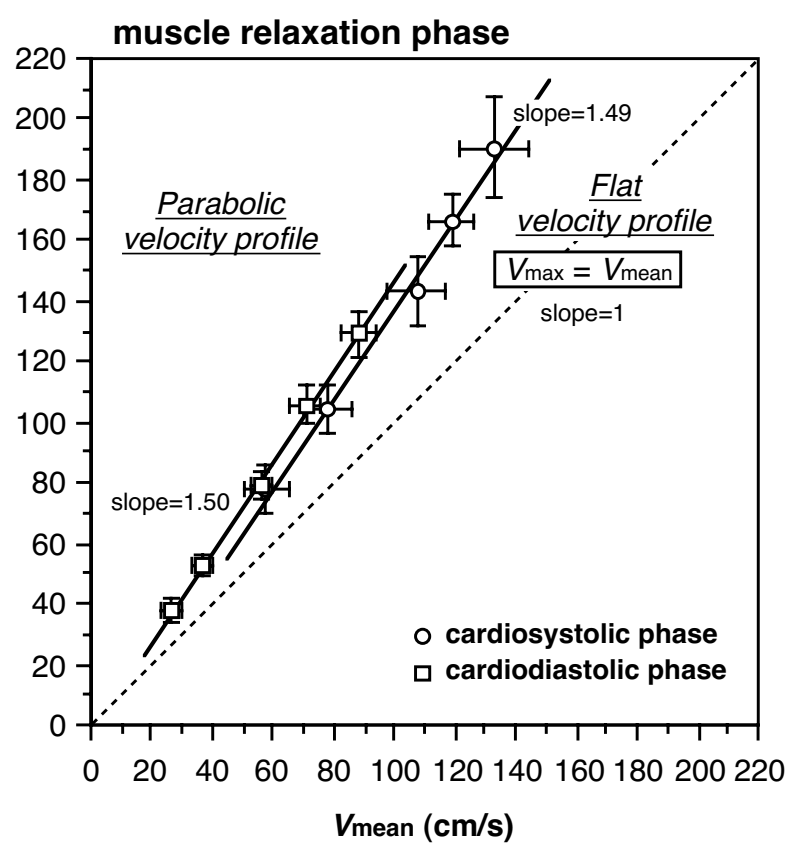

cardiosystolic (1.74, solid circles) and cardiodiastolic (2.48, solid squares) phases ( $\leftrightarrow$ in the figure). However, during muscle relaxation the slope of the regression line was "similar" $(p=$ NS) for the cardiosystolic (1.49, open circles) and cardiodiastolic (1.50, open squares) phases. The steepness of the parabolic blood velocity profile shifted in a somewhat more "flattening" direction during muscle relaxation. The values are expressed as mean \pm SE.

phase the $V_{\text {max }} / V_{\text {mean }}$ ratio increased $(p<0.05)$ somewhat to a $V_{\text {max }} / V_{\text {mean }}$ ratio of 1.54 (Fig. 2). Although the velocity profile at rest showed a steeper parabolic-like pattern during the cardiodiastolic phase than during the cardiosystolic phase $[2,4,5,11,15,30]$, there was no difference in the $V_{\max } / V_{\text {mean }}$ ratio between the cardiosystolic and cardiodiastolic phase during the muscle relaxation phase of dynamic exercise. A more flat-like velocity profile was found during the cardiosystolic phase during both the muscle contraction and muscle relaxation phases (Fig. 4). Further, the slope during the cardiodiastolic phase was similar to that during the cardiosystolic phase during the muscle relaxation phase, but not during the muscle contraction phase. Therefore the flattening of the blood velocity profile during dynamic exercise was enhanced during the muscle relaxation phase, and especially as it coincided with the "cardiodiastolic phase."

Moreover, the blood velocity distribution of "oscillating" (pulsatile) flow in a straight cylindrical elastic tube has previously been assumed to be related to a single nondimensional parameter $\alpha$ (Womersley parameter) [31, 32], described by the formula where $\alpha=D / 2 \times \sqrt{(2 \pi \rho n / \mu)}$ $(D$, diameter; $\rho$, density; $n$, flow frequency; $\mu$, viscosity). The oscillating-boundary-layer length corresponds to $\sqrt{(2 \pi \rho n / \mu)}$. The parameter $\alpha$ is thus influenced by both the diameter $(D)$ and the flow frequency $(n)$. In other words, 
the blood flow velocity profile may be disturbed when the parameter $\alpha$ shows a lower value, such as for small tube diameters or low pulsatile flow frequency. Even though extrapolation to the present human model may be difficult, the difference in the blood flow velocity profile between the muscle contraction and muscle relaxation phase at various work rates may potentially be influenced by the alterations in heart rate (flow frequency; internal factor) and repetitive compressions of the conduit femoral artery by the muscle contraction force (external factor) distal to the site of measurement. Thus one possible explanation for the alterations in the blood velocity profile at the different work rates during the cardiosystolic and cardiodiastolic phases for the muscle contraction and the muscle relaxation phases may be explained in accordance with the Womersley parameter because of the changes in intramuscular pressure and its relation to the state of vasodilatation, heart rate, cardiac cycle, and its effect on perfusion pressure.

\section{FBF during the cardiosystolic and cardiodiastolic phases during the muscle contraction and muscle relaxation phases}

A positive linear relationship between leg blood flow, averaged over consecutive contraction-relaxation duty cycles, and work intensity during DKE at $60 \mathrm{cpm}$ has previously been demonstrated with Doppler ultrasound $[8,9$, $11,14]$. In the present study, a strong positive linear correlation was found between FBF and work rate for both the cardiosystolic and cardiodiastolic phases during muscle relaxation, but not during muscle contraction (Fig. 3). A significant increase in both mean and maximum FBF values was also seen during both the cardiosystolic and cardiodiastolic phases during muscle relaxation, but not during muscle contraction. The present data thus support that the muscle relaxation phase, as compared to the muscle contraction phase, contributes to a much greater extent to the exercise-induced hyperemic response [10]. Femoral artery blood flow was thus higher during muscle relaxation during both the cardiosystolic and cardiodiastolic phases, i.e., when the blood velocity profile was less steep and somewhat more flat-like, possibly resulting from a lower intramuscular pressure and lower mechanical hindrance. The study consequently suggests that part of the magnitude of the hyperaemic response may be due to an alteration in the blood velocity flow profile, where a flatter velocity profile may exhibit less energy loss than a steeper parabolic velocity profile and consequently render a higher blood flow. Furthermore, the BP increase with an increase in work rate at higher exercise intensities during DKE may in turn influence the blood flow response.

Moreover, at rest the present findings are in agreement with previous findings $[2,4,5,15,30]$ showing a large upward (positive) flow velocity component during both the cardiosystolic and cardiodiastolic phases, and a small re- verse retrograde (negative) flow velocity component between the end of the cardiosystolic phase and the onset of the cardiodiastolic phase. Further, the upward (positive) flow velocity component at rest showed a less parabolic and somewhat more flat-like profile during the cardiosystolic phase compared to during the cardiodiastolic phase. A somewhat more steep parabolic-like velocity profile was seen during the cardiodiastolic phase at rest because of a relatively higher blood velocity found in the center of the vessel with lower velocities near the vascular walls.

However, during rhythmic muscle contractions, the reverse retrograde negative flow velocity component (downward arrow $\downarrow$ in Fig. 1) was clearly emphasized during the muscle contraction phase because the cardiodiastolic phase coincided with low BP and high muscle contraction forces, and during the cardiosystolic phase when the intramuscular pressure was higher than the systolic BP. The increase in negative back flow velocity observed below the "zero baseline," enhanced by the high intramuscular pressure during muscle contractions, may further influence the blood velocity flow profile at high muscle contraction forces (above $30 \mathrm{~W}$ ). Consequently, the formation of a "steeper" parabolic-like blood velocity profile at higher intensities such as 30 and $40 \mathrm{~W}$, which occurs during the cardiosystolic and cardiodiastolic phases during muscle contraction (Fig. 2), influenced by the higher intramuscular pressure variations, may in turn decrease the magnitude of the blood flow increase as a result of exercise during the contraction phase. On the contrary, the blood velocity profile during muscle relaxation represented by an unimpeded upward positive flow velocity component, showed less of a modification and remained at a more flat-like velocity profile, inducing a larger blood flow response to exercise during the relaxation phase.

\section{CONCLUSION}

The major finding of the present study was the observation of an alteration in the blood velocity profile in response to rhythmic muscle contractions at different exercise intensities during the muscle contraction and muscle relaxation phases in relation to the cardiac cycle. The "steepness" of the parabolic velocity profile increased during incremental exercise, specifically when the muscle contraction phase coincided with the cardiodiastolic phase. The "steepness" of the profile during the cardiosystolic phase, during both the muscle contraction and relaxation phases, increased only for the two higher muscle contraction intensities. In contrast, the velocity profile shifted to be less steep and more flat-like during the cardiodiastolic phase in the muscle relaxation phase in comparison to rest. The blood velocity profile thus appeared to be influenced by the muscle contraction-induced mechanical hindrance, along with the temporal influence of the cardiac cycle. The higher magnitude in the blood flow 
response during muscle relaxation, during both the cardiosystolic and the cardiodiastolic phases, may consequently be partly due to the less parabolic and somewhat more flat-like velocity profile. Thus the blood velocity profile may have a direct effect on the blood flow response to exercise. This is furthermore of great importance as various states of cardiovascular disease additionally may alter the blood velocity profile and further disturb the blood flow response to exercise, ultimately limiting performance.

The authors wish to acknowledge the support given by Professor Bengt Saltin, the staff of The Copenhagen Muscle Research Centre, and the Department of Preventive Medicine and Public Health at Tokyo Medical University, as well as the volunteers who participated in the study. The study was supported by a grant from the Danish National Research Foundation (\#504-14), partly by the "Academic Frontier" Project for Private Universities (JWCPE), and by the Hippocratic Award at Tokyo Medical University in 2000 (T. Osada).

\section{REFERENCES}

1. Hale JF, McDonald DA, Womersley JR. Velocity profiles of oscillating arterial flow, with some calculations of viscous drag and the Reynolds numbers. J Physiol (Lond). 1955;128:629-40.

2. Noordergraaf A. Circulatory System Dynamics. New York: Academic Press; 1978.

3. Sheldon CD, Murie JA, Quin RO. Ultrasonic Doppler spectral broadening in the diagnosis of internal carotid artery stenosis. Ultrasound Med Biol. 1983;9:57580.

4. Burns PN, Jaffe CC. Quantitative flow measurements with Doppler ultrasound: techniques, accuracy, and limitations. Radiol Clin North Am. 1985;23:641-57.

5. Hatle L, Angelsen B. Doppler Ultrasound in Cardiology: Physical Principles and Clinical Applications. 2nd edition. Phyladelphia: Lea \& Febiger; 1985.

6. Bishop JJ, Nance PR, Popel AS, Intaglietta M, Johnson PC. Effect of erythrocyte aggregation on velocity profiles in venules. Am J Physiol Heart Circ Physiol. 2001;280:H222-36.

7. Andersen P, Saltin B. Maximal perfusion of skeletal muscle in man. J Physiol (Lond). 1985;366:233-49.

8. Rådegran G. Ultrasound Doppler estimates of femoral artery blood flow during dynamic knee extensor exercise in humans. J Appl Physiol. 1997;83:1383-8.

9. Osada T, Rådegran G. Femoral artery inflow in relation to external and total work rate at different knee extensor contraction rates. J Appl Physiol. 2002;92:132530.

10. Rådegran $G$, Saltin $B$. Muscle blood flow at onset of dynamic exercise in humans. Am J Physiol Heart Circ Physiol. 1998;274:H314-22.

11. Osada $T$, Rådegran $G$. Alterations in the rheological flow profile in conduit femoral artery during rhythmic thigh muscle contractions in humans. Jpn J Physiol. 2005;55:19-28.

12. Andersen P, Adams RP, Sjøgaard G, Thorboe A, Saltin B. Dynamic knee extension as model for study of isolated exercising muscle in humans. J Appl Physiol. 1985;59:1647-53.
13. Sjøgaard G, Kiens B, Jørgensen K, Saltin B. Intramuscular pressure, EMG and blood flow during low-level prolonged static contraction in man. Acta Physiol Scand. 1986;128:475-84.

14. Osada T. Muscle contraction-induced limb blood flow variability during dynamic knee extensor. Med Sci Sports Exerc. 2004;36:1149-58.

15. Whitmore RC. Rheology of the Circulation. Oxford: Pergamon Press; 1968.

16. McDonald DA. The velocity profile in pulsatile flow. In: Blood Flow in Arteries. 2nd edition. London: Edward Arnold; 1974. p. 101-17.

17. Sugawara M. Hemodynamics (Chapter 3). In: Handbook of Physiological Sciences, Cardiovascular Physiology. Irisawa H, Kumada M, editors. Tokyo: Igaku-Shoin Ltd.; 1991. Vol 16, p. 198-216.

18. Maeda N. Erythrocyte rheology in microcirculation. Jpn J Physiol. 1996;46:1-14.

19. Shoemaker JK, Hodge L, Hughson RL. Cardiorespiratory kinetics and femoral artery blood velocity during dynamic knee extension exercise. J Appl Physiol. 1994;77:2625-32.

20. Wesche J. The time course and magnitude of blood flow changes in the human quadriceps muscles following isometric contraction. J Physiol (Lond). 1986;377:445-62.

21. Leyk D, Eßfeld D, Baum K, Stegemann J. Influence of calf muscle contractions on blood flow parameters measured in the arteria femoralis. Int J Sports Med. 1992;13:588-93.

22. Isnard R, Lechat P, Kalotka H, Chikr H, Fitoussi S, Salloum J, Golmard J-L, Thomas D, Komajda M. Muscular blood flow response to submaximal leg exercise in normal subjects and in patients with heart failure. J Appl Physiol. 1996;81:2571-9.

23. Osada T, Katsumura T, Hamaoka T, Inoue S, Esaki K, Sakamoto A, Murase N, Kajiyama J, Shimomitsu T, Iwane H. Reduced blood flow in abdominal viscera measured by Doppler ultrasound during one-legged knee extension. J Appl Physiol. 1999;86:709-19.

24. Osada T, Katsumura T, Hamaoka T, Murase N, Naka M, Shimomitsu T. Quantitative effects of respiration on venous return during single knee extensionflexion. Int J Sports Med. 2002;23:183-90.

25. Osada T, Katsumura T, Murase N, Sako T, Higuchi H, Kime R, Hamaoka T, Shimomitsu T. Post-exercise hyperemia after ischemic and non-ischemic isometric handgrip exercise. J Physiol Anthropol Appl Human Sci. 2003;22:299309.

26. Osada T. Exercise-related time course of pulsatility index in brachial artery following forearm exercise assessed by Doppler ultrasound. Tohoku J Exp Med. 2004;203:241-52.

27. Gill RW. Measurement of blood flow by ultrasound: Accuracy and sources of error. Ultrasound Med Biol. 1985;11:625-41.

28. Hughson RL, MacDonald MJ, Shoemaker JK, Borkhoff C. Alveolar oxygen uptake and blood flow dynamics in knee extension ergometry. Methods Inf Med. 1997;36:364-7.

29. MacDonald MJ, Shoemaker JK, Tschakovsky ME, Hughson RL. Alveolar oxygen uptake and femoral artery blood flow dynamics in upright and supine leg exercise in humans. J Appl Physiol. 1998;85:1622-8.

30. Lunt MJ, Jenkinson DF, Kerr D. Transcranial Doppler blood velocity measurement-The effect of changes in velocity profile. Ultrasound Med Biol. 2000;26:1145-51.

31. Womersley JR. Oscillatory motion of a viscous liquid in a thin-walled elastic tubeI: The linear approximation for long waves. Phil Mag. 1955;46:199-221.

32. Womersley JR. Oscillatory flow in arteries: the constrained elastic tube as a model of arterial flow and pulse transmission. Phys Med Biol. 1957;2:178-87. 